\title{
On the Conditions for Existence of Unidirectional Motions of Binary Mixtures in the Oberbeck-Boussinesq Model
}

\author{
V. K. Andreev ${ }^{1,2^{*}}$ and I. V. Stepanova ${ }^{1,2^{* *}}$ \\ ${ }^{1}$ Institute of Computational Modeling, Akademgorodok 50/44, Krasnoyarsk, 660036 Russia \\ ${ }^{2}$ Institute of Mathematics and Fundamental Informatics, Siberian Federal University, \\ pr. Svobodnyi 79, Krasnoyarsk, 660036 Russia
}

Received December 24, 2018; in final form, December 24, 2018; accepted March 14, 2019

\begin{abstract}
Compatibility conditions are obtained for the nonstationary Oberbeck-Boussinesq equations describing the unidirectional motions of a liquid binary mixture in a horizontal strip. We examine the case of polynomial dependence of temperature on the longitudinal coordinate is considered, and the influence of the dependence of the kind on the remaining unknown functions from the original system. It is shown that a nonstationary unidirectional motion between two solid walls can be described by the Oberbeck-Boussinesq model only for the quadratic or linear law of temperature distribution along the horizontal coordinate. Some initial-boundary value problems are posed.
\end{abstract}

\section{DOI: $10.1134 / \mathrm{S} 1990478919020017$}

Keywords: Oberbeck-Boussinesq equations, binary mixture, unidirectional motion, compatibility conditions

\section{INTRODUCTION}

The Oberbeck-Boussinesq equations often form the basis of a mathematical model of convective motion of a liquid in the natural environment. To study the effect of thermal diffusion on the distribution of components of a binary mixture, it is necessary to supplement the described model with the diffusion equation. These equations are asymmetric with respect to the unknown functions: The thermal diffusion effect is taken into account in the equation for concentration by an extra term related to temperature. The resultant system is nonlinear and has high order. The construction of exact solutions for such systems is a complex and important problem of modern mathematics and its applications in continuum mechanics.

We should note that sufficiently many exact solutions are known for the model of stationary convection in a homogeneous liquid (see, for example, the monograph [1] and survey [2]). The results of studying the equations of thermal diffusion convection can be found in [3, 4]. Some problems concerning the stability of thermal diffusion flows as well as the mutual influence of thermal diffusion and vibrational impact on mixtures are described in [5,6]. The monograph [7] is devoted to construction and study of solutions of nonstationary problems of thermal diffusion in plane and cylindrical domains as well as to derivation of some sufficient conditions for their convergence to a steady state.

The study in this article is an extension and generalization of the results of [8-10]. In particular, in [8] the compatibility analysis and construction of the new exact solutions were carried out for the equations of unidirectional steady flow of a binary mixture with nonlinear buoyancy force and the density being an arbitrary function of temperature and concentration. The article [9] contains the most detailed investigation of these flows with a linear dependence of density on the state parameters. In [10], it is shown that the unidirectional motion of a homogeneous liquid in the classical Oberbeck-Boussinesq model exists only under a linear dependence of temperature on the longitudinal coordinate along the liquid layer. Moreover, the temperature gradient on the layer boundaries may depend on time.

\footnotetext{
${ }^{*}$ E-mail: andr@icm.krasn.ru

${ }^{* *}$ E-mail: stepiv@icm.krasn.ru
} 
The article is devoted to the analysis of nonstationary equations for the unidirectional motion of a binary mixture in the Oberbeck-Boussinesq approximation. The compatibility condition for this system is reduced to the fact that the temperature function must satisfy Poisson's equation with the right-hand side linear in the horizontal coordinate $x$, whereas the concentration function should have the same character of dependence on $x$ as the temperature. The obtained results provide new information about unsteady motions of binary mixtures and generalize the analysis of [10] which was carried out for homogeneous liquids.

\section{COMPATIBILITY ANALYSIS OF THE EQUATIONS FOR UNIDIRECTIONAL UNSTEADY MOTIONS OF A BINARY MIXTURE}

The equations of unsteady unidirectional motion of a binary mixture are as follows:

$$
\begin{gathered}
u_{t}=\nu u_{y y}-\frac{1}{\rho_{0}} p_{x}^{*}, \quad g\left(\beta_{1} \theta+\beta_{2} c\right)=\frac{1}{\rho_{0}} p_{y}^{*}, \\
\theta_{t}+u \theta_{x}=\chi\left(\theta_{x x}+\theta_{y y}\right), \quad c_{t}+u c_{x}=D\left(c_{x x}+c_{y y}\right)+D^{\theta}\left(\theta_{x x}+\theta_{y y}\right),
\end{gathered}
$$

where $u=u(y, t)$ is the horizontal component of velocity, $\theta=\theta(x, y, t)$ is the temperature, $c=c(x, y, t)$ is the concentration of the light component, and $p^{*}=p-g \rho_{0} y$ is the modified pressure. The constants $\nu>0, \chi>0, D>0$, and $D^{\theta}$ are the coefficients of kinematic viscosity, temperature conductivity, diffusion, and thermal diffusion respectively. System (1) describes the motion of a binary mixture in the Oberbeck-Boussinesq approximation; i.e., the equation of state has the form $\rho=\rho_{0}\left(1-\beta_{1} \theta-\beta_{2} c\right)$, where $\beta_{1}$ and $\beta_{2}$ are the coefficients of thermal and concentration expansion, and $\rho_{0}$ is the average density of the mixture.

Taking $L$ as the characteristic length scale and $\Delta T$, as the characteristic temperature, we introduce the dimensionless variables

$$
\begin{gathered}
\hat{x}=\frac{x}{L}, \quad \hat{y}=\frac{y}{L}, \quad \hat{t}=\frac{\nu}{L^{2}} t, \quad \hat{u}=\frac{\nu}{g \beta_{1} \Delta T L^{2}} u, \\
\hat{p}^{*}=\frac{1}{\rho_{0} g \beta_{1} \Delta T L} p^{*}, \quad \hat{\theta}=\frac{1}{\Delta T} \theta, \quad \hat{c}=\frac{\beta_{2}}{\beta_{1} \Delta T} c .
\end{gathered}
$$

In these variables, system (1) has the form (the "hat" is omitted)

$$
\begin{gathered}
u_{t}=u_{y y}-p_{x}, \quad \theta+c=p_{y}, \\
\theta_{t}+\operatorname{Gr} u \theta_{x}=\frac{1}{\operatorname{Pr}}\left(\theta_{x x}+\theta_{y y}\right), \quad c_{t}+\operatorname{Gr} u c_{x}=\frac{1}{\operatorname{Sc}}\left[c_{x x}+c_{y y}-\psi\left(\theta_{x x}+\theta_{y y}\right)\right],
\end{gathered}
$$

where $\mathrm{Gr}=g \beta_{1} \Delta T L^{3} / \nu^{2}$ is the Grashof number, $\operatorname{Pr}=\nu / \chi$ is the Prandtl number, $\mathrm{Sc}=\nu / D$ is the Schmidt number, and $\psi=-\beta_{2} D^{\theta} /\left(\beta_{1} D\right)$ is the separation ratio.

The first equation of $(2)$ yields the linearity of the pressure in $x$

$$
p(x, y, t)=-a(y, t) x+b(y, t),
$$

whereas the second one, the condition on the temperature and concentration functions

$$
\theta+c=b_{y}-a_{y} x
$$

In (3) and (4), the functions $a(y, t)$ and $b(y, t)$ are still arbitrary. Expressing in (4) the concentration through temperature and inserting the results into the last equation of (2), after simple transformations we arrive at another equation on $\theta(x, y, t)$ :

$$
\theta_{t}+\operatorname{Gr} u \theta_{x}=\frac{1+\psi}{\mathrm{Sc}}\left(\theta_{x x}+\theta_{y y}\right)+(b-a x)_{t y}-\frac{1}{\mathrm{Sc}}(b-a x)_{y y y}-\operatorname{Gr} u a_{y} .
$$

Comparing (5) and the third equation of (2), we obtain

$$
\left[\frac{1}{\mathrm{Pr}}-\frac{1+\psi}{\mathrm{Sc}}\right]\left(\theta_{x x}+\theta_{y y}\right)=(b-a x)_{t y}-\frac{1}{\mathrm{Sc}}(b-a x)_{y y y}-\mathrm{Gr} u a_{y} .
$$


For further reasoning, it is convenient to introduce the parameter

$$
\Psi=1 / \operatorname{Pr}-(1+\psi) / \mathrm{Sc} .
$$

Note that $\Psi=0$ provided the physical properties of the mixture satisfy $\chi+D^{\theta} \beta_{2} / \beta_{1}=D$. In general, $\Psi \neq 0$. Then it follows from (6) that $\theta_{x x}+\theta_{y y}$ is a linear function of $x$ :

$$
\theta_{x x}+\theta_{y y}=\frac{1}{\Psi}\left[\left(\frac{1}{\mathrm{Sc}} a_{y y y}-a_{t y}\right) x+b_{t y}-\frac{1}{\mathrm{Sc}} b_{y y y}-\mathrm{Gr} u a_{y}\right] .
$$

Thus, the temperature satisfies the inhomogeneous linear partial differential equation of the first order:

$$
\theta_{t}+\operatorname{Gr} u \theta_{x}=\frac{1}{\operatorname{Pr} \Psi}\left[\left(\frac{1}{\mathrm{Sc}} a_{y y}-a_{t}\right)_{y} x+\left(b_{t}-\frac{1}{\mathrm{Sc}} b_{y y}\right)_{y}-\operatorname{Gr} u a_{y}\right] \equiv \alpha(y, t) x+\beta(y, t) .
$$

If $\theta_{0}(x, y)$ is the initial temperature distribution then the solution of $(9)$ is given as

$$
\begin{aligned}
\theta(x, y, t)=\theta_{0}\left(x-\operatorname{Gr} \int_{0}^{t} u(y, \tau) d \tau, y\right) & \\
& +\int_{0}^{t}\left\{\alpha(y, \tau)\left[x+\operatorname{Gr} \int_{0}^{\tau} u\left(y, \tau_{1}\right) d \tau_{1}\right]+\beta(y, \tau)\right\} d \tau \\
& -\operatorname{Gr} \int_{0}^{t} \alpha(y, \tau) d \tau \int_{0}^{t} u(y, \tau) d \tau .
\end{aligned}
$$

The field of concentrations is obtained from (4)

$$
c(x, y, t)=b_{y}-a_{y} x-\theta(x, y, t), \quad c_{0}(x, y)=b_{y}(y, 0)-a_{y}(y, 0) x-\theta_{0}(x, y)
$$

with the function $\theta(x, y, t)$ from (10).

Note that the temperature distribution $\theta_{0}$ is not arbitrary, but must satisfy the Poisson's equation (see $(8))$ :

$$
\theta_{0 x x}+\theta_{0 y y}=\operatorname{Pr}[\alpha(y, 0) x+\beta(y, 0)]
$$

with the right-hand side linear in $x$.

Inserting $\theta$ from (10) into (8) for $t \geq 0$, we arrive at the relation

$$
\begin{array}{r}
\theta_{0 \xi \xi}\left[1+\mathrm{Gr}^{2}\left(\int_{0}^{t} u_{y} d \tau\right)^{2}\right]-2 \operatorname{Gr} \operatorname{theta}_{0 \xi y} \int_{0}^{t} u_{y} d \tau+\theta_{0 y y}-\operatorname{Gr} \theta_{0 \xi} \int_{0}^{t} u_{y y} d \tau \\
=\operatorname{Pr}[\alpha(y, t) x+\beta(y, t)]-Q_{y y}(y, t) x-Q_{1 y y}(y, t)
\end{array}
$$

where

$$
\begin{gathered}
\xi=x-\operatorname{Gr} \int_{0}^{t} u(y, \tau) d \tau, \quad Q(y, t)=\int_{0}^{t} \alpha(y, \tau) d \tau \\
Q_{1}(y, t)=\int_{0}^{t}\left[\operatorname{Gr} \alpha(y, \tau) \int_{0}^{\tau} u\left(y, \tau_{1}\right) d \tau_{1}+\beta(y, \tau)\right] d \tau-\operatorname{Gr} \int_{0}^{t} \alpha(y, \tau) d \tau \int_{0}^{t} u(y, \tau) d \tau .
\end{gathered}
$$

This reasoning allows us to make the following conclusions:

1. If the given initial temperature $\theta_{0}(x, y)$ is consistent with (12) then the initial data $\alpha(y, 0)$ and $\beta(y, 0)$ will be known. In this case (13) with notation (14) defines the connections between the functions 
$u(y, t), \alpha(y, t)$, and $\beta(y, t)$. To make the problem closed, it is necessary to supplement these relations with the first equation of (2) taking (3) into account:

$$
u_{t}=u_{y y}+a(y, t) .
$$

2. In general, the form of the function $\theta_{0}(x, y)$, which is the solution of (12), depends on the type of boundary conditions, for example, on the solid walls $y=0$ and $y=1$. Since the horizontal velocity is only a function of $y$; therefore, it is impossible to satisfy the no-slip boundary conditions for finite $x$; hence, the problem makes sense only in a layer unbounded with respect to $x$.

3 . While constructing exact solutions, the temperature and concentration are often taken in the form of polynomials of the second $[2,7,9,11,12]$ or first $[2-4,13-17]$ degree in the horizontal coordinate $x$. In our case, due to the fact that $\theta_{0}(x, y)$ must satisfy (12), the representation of $\theta_{0}(x, y)$ in polynomial form can be a polynomial in $x$ of the degree at most 3 .

\section{POLYNOMIAL TEMPERATURE DISTRIBUTION WITH RESPECT TO THE HORIZONTAL COORDINATE} that

It was noted that $\theta_{0}(x, y)$, and so $\theta(t, x, y)$, can be a polynomial in $x$ of the degree at most 3 . Suppose

$$
\theta=s_{1}(y, t) x^{3}+s_{2}(y, t) x^{2}+s_{3}(y, t) x+s_{4}(y, t)
$$

with still arbitrary functions $s_{i}, i=1, \ldots, 4$. Then it follows from (4) that the representation for the concentration must be of the form

$$
c=s_{5}(y, t) x^{3}+s_{6}(y, t) x^{2}+s_{7}(y, t) x+s_{8}(y, t),
$$

whereas $s_{5}=-s_{1}, s_{6}=-s_{2}, s_{7}=-s_{3}-a_{y}$, and $s_{8}=b_{y}-s_{4}$. Inserting a solution of this type into the last two equations of (2) and subsequently splitting with respect to the powers of $x$ (taking $\Psi \neq 0$ into account) leads to the following relations for $s_{i}, i=1, \ldots, 8$ :

$$
\begin{aligned}
& s_{1 t}=0, \quad s_{1 y y}=0, \quad s_{2 y y}=0, \quad s_{2 t}=-3 \operatorname{Gr} u s_{1}, \\
& s_{3 t}+2 \mathrm{Gr} u s_{2}=\frac{1}{\operatorname{Pr}}\left(6 s_{1}+s_{3 y y}\right), \quad s_{4 t}+2 \mathrm{Gr} u s_{3}=\frac{1}{\operatorname{Pr}}\left(2 s_{2}+s_{4 y y}\right), \\
& s_{7 t}-2 \mathrm{Gr} u s_{2}=\frac{1}{\mathrm{Sc}}\left(s_{7 y y}-\psi s_{3 y y}-6(1+\psi) s_{1}\right), \\
& s_{8 t}+\operatorname{Gr} u s_{7}=\frac{1}{\mathrm{Sc}}\left(s_{8 y y}-\psi s_{4 y y}-2(1+\psi) s_{2}\right) .
\end{aligned}
$$

Equation (15) for $u$ remains in the same form. For the so-obtained $s_{i}$, the functions $a$ and $b$ are reconstructed from the equalities

$$
a_{y}=-s_{7}-s_{3}, \quad b_{y}=s_{4}+s_{8} .
$$

From the first four equalities in (18) it follows that if $s_{1}=\varphi_{1} y+\varphi_{2} \neq 0$ and $s_{2}=\varphi_{3}(t) y+\varphi_{4}(t)\left(\varphi_{1}\right.$ and $\varphi_{2}$ are constants, while $\varphi_{3}$ and $\varphi_{4}$ are functions of $t$ ) then the function $u$ has the special form

$$
u=-\frac{1}{3 \mathrm{Gr}} \frac{\varphi_{3}^{\prime} y+\varphi_{4}^{\prime}}{\varphi_{1} y+\varphi_{2}} .
$$

The prime stands for derivative with respect to $t$. This dependence of the velocity restricts the options of setting the boundary conditions for the determination of the velocity from (15). For example, the no-slip conditions on two fixed solid walls cannot be satisfied. Similarly, the fulfillment of the thermal insulation condition on one of the walls, for example, the upper one $(\partial \theta(x, 1, t) / \partial y=0)$, implies $\varphi_{1}=\varphi_{3}(t)=0$; i.e, the velocity function does not depend on $y$. As another example of the impossibility of using (20) to describe the motion, we refer to the condition of absence of the substance flow, say, on the lower wall $y=0$ :

$$
\frac{\partial c}{\partial y}(x, 0, t)-\psi \frac{\partial \theta}{\partial y}(x, 0, t)=0
$$


Inserting the representations (16) and (17) into (21) and splitting the resultant equalities with respect to $x$, we note that $(1+\psi) \varphi_{1}=0$ and $(1+\psi) \varphi_{3}(t)=0$; whence, in the case of $\psi \neq-1$, it follows that the velocity does not depend on $y$.

In the case of $\psi=-1$, the density gradients, caused by the temperature and concentration inhomogeneity, balance each other. The density becomes constant, and equations (1) go beyond the framework of the Oberbeck-Boussinesq model. Sometimes, instead of $(21)$, the zero concentration $c(x, 0, t)=0$ is given. Then $\varphi_{2}=0$ and $\varphi_{4}(t)=0$; and again the velocity determined by (20) is a function of only $t$.

Thus, the following conclusion is valid:

4. In the general case ( $\Psi \neq 0$ and $\psi \neq-1$ ), the unidirectional motion of a binary mixture on a solid wall is impossible if the temperature and concentration are third-degree polynomials.

The same conclusion can be drawn if we consider the line $y=1$ as the free boundary on which the dynamic condition $\rho_{0} \nu u_{y}=-\varkappa_{1} \theta_{x}-\varkappa_{2} c_{x}$ is given, where $\varkappa_{1}$ is the thermal coefficient of surface tension, $\varkappa_{2}$ is the coefficient of surface activity of the free boundary. In the dimensionless variables, this condition will be rewritten as

$$
\left.\left(u_{y}+\frac{\mathrm{Ma}^{\theta}}{\mathrm{Gr}} \theta_{x}+\frac{\mathrm{Ma}^{c}}{\mathrm{Gr}} c_{x}\right)\right|_{y=1}=0 .
$$

Here, $\mathrm{Ma}^{\theta}=\varkappa_{1} \triangle T L /\left(\rho_{0} \nu^{2}\right)$ and $\mathrm{Ma}^{c}=\varkappa_{2} \beta_{1} \triangle T L /\left(\beta_{2} \rho_{0} \nu^{2}\right)$ are the thermal and concentration Marangoni numbers (the dimensionless parameters). Inserting the form of the functions $u, \theta$, and $c$ from (20), (16), and (17), we see that, after separation with respect to the powers of $x$, the condition on the free boundary splits into the equalities

$$
\begin{gathered}
s_{1}\left(\mathrm{Ma}^{\theta}-\mathrm{Ma}^{c}\right)=0, \quad s_{2}\left(\mathrm{Ma}^{\theta}-\mathrm{Ma}^{c}\right)=0, \\
\left(\frac{\varphi_{3}^{\prime} y+\varphi_{4}^{\prime}}{\varphi_{1} y+\varphi_{2}}\right)_{y}-3\left(\mathrm{Ma}^{\theta} s_{3}-\mathrm{Ma}^{c} s_{7}\right)=0,
\end{gathered}
$$

from which it follows that the cubic dependence of the temperature and concentration $\left(s_{1} \neq 0\right)$ is possible only in the mixtures satisfying $\varkappa_{1} / \beta_{1}=\varkappa_{2} / \beta_{2}$. In general, conclusion 4 is valid for the motion of the mixture on a solid wall with a free upper boundary.

Let us turn to the analysis of the quadratic dependence of $\theta$ and $c$ on $x$. In the case of the steady temperature distribution $\theta=s_{2}(y) x^{2}+s_{3}(y) x+s_{4}(y)$, the functions $s_{i}, i=2,3,4,7,8$, are found in the authors' article [8]. The formulations of boundary value problems are also proposed there to describe the steady liquid flow in a channel with solid walls and free surface with the given dependences of temperature and concentration. Note also that, in the case of a joint two-layer flow of a binary mixture and a homogeneous liquid, the quadratic temperature dependence is used in [11,12] to describe thermalconcentration convection with the dependence of the velocity on two spatial coordinates.

Put $s_{1} \equiv 0\left(s_{5} \equiv 0\right)$ in (16) and (17). Then $s_{2}=\varphi_{5} y+\varphi_{6}$ with the constant $\varphi_{5}$ and $\varphi_{6}$. By analogy to the analysis of cubic dependence, in the general case of $\Psi \neq 0$ and $\psi \neq-1$, we can assume that $\varphi_{5}=0$, whereas $\varphi_{6}$ is some given constant. Taking the first equality in (19) into account, we deduce from (15) the equation for the velocity

$$
u_{t}-u_{y y}=-\int_{y_{0}}^{y}\left[s_{3}(z, t)+s_{7}(z, t)\right] d z+f(t)
$$

with an arbitrary function $f(t)$.

Remark 1. According to (3), the right-hand side of (22) taken with the opposite sign is the pressure gradient along the $x$-axis. Thus, only the coefficients at the first degree of $x$ in (16) and (17) affect the motion of the liquid, as well as the function $f(t)$ which can be called an additional pressure gradient. 
Remark 2. Since $s_{2}=\varphi_{6}=$ const, the equations on $s_{3}(y, t), s_{7}(y, t)$, and $u(y, t)$ form the closed system of linear equations

$$
\begin{gathered}
s_{3 t}+2 \operatorname{Gr} u \varphi_{6}=\frac{1}{\operatorname{Pr}} s_{3 y y}, \quad s_{7 t}-2 \operatorname{Gr} u \varphi_{6}=\frac{1}{\operatorname{Sc}}\left(s_{7 y y}-\psi s_{3 y y}\right), \\
u_{t}-u_{y y}=-\int_{y_{0}}^{y}\left[s_{3}(z, t)+s_{7}(z, t)\right] d z+f(t) .
\end{gathered}
$$

The functions $s_{4}(y, t)$ and $s_{8}(y, t)$ are found from the two parabolic equations

$$
s_{4 t}+2 \operatorname{Gr} u s_{3}=\frac{1}{\operatorname{Pr}}\left(2 \varphi_{6}+s_{4 y y}\right), \quad s_{8 t}+\operatorname{Gr} u s_{7}=\frac{1}{\mathrm{Sc}}\left[s_{8 y y}-\psi s_{4 y y}-2(1+\psi) \varphi_{6}\right] .
$$

For system (23), (24) it is possible to formulate various initial-boundary value problems that describe certain motions of the mixture in a flat layer. Let us present one of the formulations describing the motion of the mixture in a layer with solid motionless walls $y=0$ and $y=1$, whereas the temperature

$$
\theta_{1}(x, t)=\varphi_{6} x^{2}+s_{31}(t) x+s_{41}(t)
$$

is given on the lower wall, while the upper wall is thermal isolated and $\theta_{y}(x, 1, t)=0$. And more, the conditions of no-slip and the absence of the substance flow in the form (21) are satisfied on these walls. Thus, we have the boundary conditions

$$
\begin{gathered}
s_{3}(0, t)=s_{31}(t), \quad s_{4}(0, t)=s_{41}(t), \quad s_{7 y}(0, t)=\psi s_{3 y}(0, t), \\
s_{8 y}(0, t)=\psi s_{4 y}(0, t), \quad u(0, t)=0 . \\
s_{3 y}(1, t)=0, \quad s_{4 y}(1, t)=0, \quad s_{7 y}(1, t)=0, \quad s_{8 y}(1, t)=0, \quad u(1, t)=0 .
\end{gathered}
$$

It is necessary to supplement (25) with the initial data

$$
s_{3}(y, 0)=s_{30}(y), \quad s_{4}(y, 0)=s_{40}(y), \quad s_{7}(y, 0)=s_{70}(y), \quad s_{8}(y, 0)=s_{80}(y)
$$

with some given functions $s_{30}(y), s_{40}(y), s_{70}(y)$, and $s_{80}(y)$ for $y \in[0,1]$.

For a smooth solution to exist, the following matching conditions for the functions included in the right-hand sides of (25) and (26) must be fulfilled:

$$
\begin{array}{cc}
s_{31}(0)=s_{30}(0), & s_{41}(0)=s_{40}(0), \\
\frac{\partial s_{70}(0)}{\partial y}=\psi \frac{\partial s_{30}(0)}{\partial y}, & \frac{\partial s_{80}(0)}{\partial y}=\psi \frac{\partial s_{40}(0)}{\partial y}, \\
\frac{\partial s_{30}(1)}{\partial y}=0, & \frac{\partial s_{40}(1)}{\partial y}=0 .
\end{array}
$$

If the function $f(t)$ from (22) is given then the statement of the problem under consideration is complete. In case $f(t)$ is unknown, we need one more condition on the velocity $u(y, t)$. Usually, this is the setting of the volumetric flow rate

$$
\int_{0}^{1} u(z, t) d z=v(t) .
$$

In this case, the problem is an inverse one. Its interpretation is as follows: What additional pressure gradient $f(t)$ should be applied to provide a given mixture flow rate $v(t)$ ?

Remark 3. The temperature on the lower wall in the form $\theta_{1}(x, t)=\varphi_{6} x^{2}+s_{31}(t) x+s_{41}(t)$ means that it has an extremum at the point $x_{0}(t)=-s_{31}(t) / 2 \varphi_{6}$ (a maximum for $\varphi_{6}<0$ and a minimum for $\left.\varphi_{6}>0\right)$.

Finally, if in addition to $s_{1} \equiv 0$ we assume that $s_{2} \equiv 0\left(\varphi_{6}=0\right)$ then the distributions of (16) and (17) will be linear in $x$. System (23), (24) is simplified, and the form of conditions (25)-(28) remains unchanged. 
Remark 4. We should note that setting the functions of the temperature and concentration in the form (16), (17) is consistent with equation (13) on $\theta$, which can be considered to be the compatibility condition of the original system (2).

Let us demonstrate this by the example of linear dependence of $\theta_{0}$ and $\theta$ on $x$. Thus, if $\theta_{0}(x, y)=$ $A_{0}(y) x+A_{1}(y)$, then we have from Poisson's equation (12)

$$
A_{0 y y}=\operatorname{Pr} \alpha(y, 0), \quad A_{1 y y}=\operatorname{Pr} \beta(y, 0) .
$$

Relation (13) yields the equality

$$
A_{1 y y}+A_{0 y y} x-\operatorname{Gr}\left[A_{0}(y) \int_{0}^{t} u(y, \tau) d \tau\right]_{y y}=\operatorname{Pr}(\alpha x+\beta)-Q_{y y} x-Q_{1 y y},
$$

which, with respect to $x$, splits into the two equations:

$$
A_{0 y y}=\operatorname{Pr} \alpha-Q_{y y}, \quad A_{1 y y}-\operatorname{Gr}\left[A_{0} \int_{0}^{t} u(y, \tau) d \tau\right]_{y y}=\operatorname{Pr} \beta-Q_{1 y y} .
$$

If we put

$$
\begin{gathered}
A_{0}(y)+\int_{0}^{t} \alpha(y, \tau) d \tau=s_{3}(y, t) \\
A_{1}(y) \int_{0}^{t}\left\{\beta(y, \tau)+\operatorname{Gr}\left[\alpha(y, \tau) \int_{0}^{\tau} u\left(y, \tau_{1}\right) d \tau_{1}-A_{0}(y) u(y, \tau)\right]\right\} d \tau \\
-\operatorname{Gr} \int_{0}^{t} \alpha(y, \tau) d \tau \int_{0}^{t} u(y, \tau) d \tau=s_{4}(y, t)
\end{gathered}
$$

then we obtain from (29) that

$$
s_{3 t}=s_{3 y y} / \operatorname{Pr}, \quad s_{4 t}=s_{4 y y} / \operatorname{Pr}-\mathrm{Gr} u s_{3} .
$$

Thus, formulas (30) show that the temperature is sought in the form

$$
\theta(x, y, t)=s_{3}(y, t) x+s_{4}(y, t) .
$$

According to (11), the concentration is also the linear function of $x$ :

$$
\begin{gathered}
c(x, y, t)=s_{7}(y, t) x+s_{8}(y, t), \\
s_{7}(y, t) x=-a_{y}(y, t)-s_{3}(y, t), \quad s_{8}(y, t)=b_{y}(y, t)-s_{4}(y, t)
\end{gathered}
$$

were $s_{i}(y, t), i=3,4,7,8$ are from (16) and (17).

\section{SOLVABILITY OF SYSTEM (2) IN THE CASE $\Psi=0$}

In the previous sections, the general case was under study: The parameter $\Psi$ in (7) was considered different from zero. If we put $\Psi$ equal to zero then the physical constants of the mixture are related by the equality $D=\chi+\beta_{2} D^{\theta} / \beta_{1}$, and the right-hand side of (6) vanishes. Therefore,

$$
\begin{gathered}
\bar{a}_{t}=\frac{1}{\mathrm{Sc}} \bar{a}_{y y}, \\
\bar{b}_{t}=\frac{1}{\mathrm{Sc}} \bar{b}_{y y}+\operatorname{Gr} u \bar{a},
\end{gathered}
$$


where

$$
\bar{a}(y, t)=a_{y}(y, t), \quad \bar{b}(y, t)=b_{y}(y, t) .
$$

In view of (33), equation (15) assumes the form

$$
u_{t}=u_{y y}+\int_{y_{0}}^{y} \bar{a}(z, t) d z+g(t)
$$

with a certain function $g(t)$.

If $g(t)$ is given then equations (31), (34) form a closed system for determining $\bar{a}(y, t)$ and $u(y, t)$. After that, the function $\bar{b}(y, t)$ not affecting the velocity field is found as the solution of (32); the temperature field, from the third equation of (2); and the concentration, from (4).

The question arises of setting the initial and boundary conditions for (31) and (32). Let $\theta_{0}(x, y)$ and $c_{0}(x, y)$ be the initial values of the temperature and concentration. Then from (4) and notation (33) we have

$$
\bar{b}(y, 0)-\bar{a}(y, 0) x=\theta_{0}(x, y)+c_{0}(x, y) \equiv q_{2}(y)-q_{1}(y) x .
$$

Hence, $\bar{a}(y, 0)=q_{1}(y)$ and $\bar{b}(y, 0)=q_{2}(y)$ are precisely the initial conditions.

The boundary conditions depend on the interpretation of the solution. Suppose that the mixture moves in a flat layer bounded by the motionless walls $y=0$ and $y=1$. On the lower wall, the heat flux is given

$$
\frac{\partial \theta}{\partial y}(x, 0, t)=G(x, t)
$$

(here $G(x, t)$ is the dimensionless heat flow), whereas the upper wall is thermal isolated:

$$
\frac{\partial \theta}{\partial y}(x, 1, t)=0 .
$$

Moreover, there is no substance flux through these walls, so that

$$
\left.\left(\frac{\partial c}{\partial y}-\psi \frac{\partial \theta}{\partial y}\right)\right|_{y=0,1}=0 .
$$

In view of (4), we derive from (35) and the first equality of (37) the dependence

$$
G(x, t)=G_{0}(t) x+G_{1}(t)
$$

with given functions $G_{0}(t)$ and $G_{1}(t)$. Turning to (35)-(37) again, we obtain the boundary conditions

$$
\bar{a}_{y}(0, t)=-(1+\psi) G_{0}(t), \quad \bar{b}_{y}(0, t)=-(1+\psi) G_{1}(t), \quad \bar{a}_{y}(1, t)=0, \quad \bar{b}_{y}(1, t)=0 .
$$

The standard initial and boundary conditions are posed for (34):

$$
u(y, 0)=u_{0}(y), \quad u(0, t)=u(1, t)=0 .
$$

If $g(t)$ is not specified then the dimensionless volumetric flow rate is taken into account according to (28). In this case, the problem of finding $u(y, t)$ and $g(t)$ is an inverse problem.

\section{CONCLUSION}

The compatibility conditions are analyzed for the Oberbeck-Boussinesq equations describing the unidirectional nonstationary motion of a binary mixture with account for the thermal diffusion effect. Some relation for the temperature function is obtained under which a solution for the original system exists. This implies that, if the temperature function is a polynomial in the powers of the horizontal variable then it can be of degree at most 3. It is shown that, in the case of the cubic dependence of temperature, it is difficult to find physically meaningful boundary conditions for velocity. Thus, although this dependence allows us to solve the original equations, the resultant solution does not seem to have any physical interpretation. For the quadratic or linear dependence of the temperature on the longitudinal 
coordinate, the equations are obtained for finding all components of the functions of the temperature, concentration, and velocity.

We propose a statement of the initial-boundary value problem for the description of motion of a mixture between two motionless solid walls. The system of equations in this problem splits into the three linear equations forming a closed subsystem and two nonlinear equations. It should be noted that the solution of the system can be obtained by the separation of variables. However, the series that form the solution converges rather slowly, for instance, if the functions $s_{3 i}(t), s_{4 i}(t), i=0,1, s_{70}(t)$, and $s_{80}(t)$

have discontinuities of the first kind. Therefore, it is more convenient to use the Laplace method for solving at least the linear part of the problem.

\section{REFERENCES}

1. G. Z. Gershuni, E. M. Zhukhovetskii, and A. A. Nepomnyashchii, Stability of Convective Flows (Nauka, Moscow, 1989) [in Russian].

2. V. K. Andreev, A Birikh Solution to the Convection Equations and Some Generalization of It, Preprint No. 1-10 (Inst. Vychisl. Mat. Model. Sibir. Otdel. Ross. Akad. Nauk, Krasnoyarsk, 2010).

3. V. K. Andreev, Yu. A. Gaponenko, O. N. Goncharova, and V. V. Pukhnachov, Mathematical Models of Convection (Fizmatlit, Moscow, 2008; Walter de Gruyter, Berlin, 2012).

4. I. I. Ryzhkov, Thermodiffusion in Mixtures: Equations, Symmetry, Solutions, and Stability (Izd. Sibir. Otdel. Ross. Akad. Nauk, Novosibirsk, 2013) [in Russian].

5. N. V. Gnevanov and B. L. Smorodin, "Convective Instability of a Binary Mixture Flow under Vibration and Thermodiffusion,” Prikl. Mekh. Tekhn. Fiz. 47 (2), 77-84 (2006).

6. I. I. Ryzhkov and I. V. Stepanova, "Group Properties and Exact Solutions to a Model of Vibrating Convection of a Binary Mixture," Prikl. Mekh. Tekhn. Fiz. 52 (4), 72-82 (2011).

7. V. K. Andreev and N. L. Sobachkina, A Binary Mixture Motion in Plane and Cylindrical Domains (Red.Izd. Tsentr of Sibir. Federal. Univ., Krasnoyarsk, 2012) [in Russian].

8. V. K. Andreev and I. V. Stepanova, "Ostroumov-Birikh Solution of Convection Equations with Nonlinear Buoyancy Force," Appl. Math. Comput. 228, 59-67 (2014).

9. V. K. Andreev and I. V. Stepanova, "Unidirectional Flow of Binary Mixtures in the Oberbeck-Boussinesq Model," Izv. Ross. Akad. Nauk. Mekh. Zhidk. Gaza No. 2, 13-24 (2016).

10. V. V. Pukhnachev, "Nonstationary Analogs of the Birikh Solution," Izv. Altaisk. Gos. Univ. Mat. Mekh. Nos. 1-2, 62-69 (2011).

11. M. V. Efimova and N. Darabi, "Thermoconcentration Convection in a System of Viscous Fluid and a Binary Mixture in a Plane Channel under Small Marangoni Numbers," Prikl. Mekh. Tekhn. Fiz. 59 (5), 93-103 (2018).

12. V. K. Andreev and M. V. Efimova, "Properties of Solutions for the Problem of a Joint Slow Motion of a Liquid and a Binary Mixture in a Two-Dimensional Channel," Sibir. J. Industr. Mat. 21 (3), 3-17 (2018) [J. Appl. Indust. Math. 12 (3), 395-408 (2018)].

13. G. A. Ostroumov, Free Convection under Conditions of Inner Problem (Gostekhizdat, Moscow, 1952) [in Russian].

14. R. V. Birikh, "On Thermocapillary Convection in a Horizontal Fluid Layer," Prikl. Mekh. Tekhn. Fiz. No. 3, 69-72 (1966).

15. O. N. Goncharova, E. V. Rezanova, Yu. V. Lyulin, and O. A. Kabov, "Studying the Convective Flows of a Fluid and Cocurrent Gas Flow with Account for Evaporation," Teplofiz. Vysokikh Temperatur 55 (6), 720$732(2017)$.

16. V. B. Bekezhanova and O. N. Goncharova, "Stability of the Exact Solutions Describing the Two-Layer Flows with Evaporation at Interface," Fluid Dynam. Res. 48 (6), 061408. (2016)

17. V. B. Bekezhanova, O. N. Goncharova, E. V. Rezanova, and I. A. Shefer, "Stability of Two-Layered Fluid Flows with Evaporation at the Interface," Izv. Ross. Akad. Nauk. Mekh. Zhidk. Gaza No. 3, 23-35 (2017). 